\title{
On the domain of a magnetic Schrödinger operator with complex electric potential.
}

\author{
B. Helffer, Laboratoire de Mathématiques Jean Leray, \\ CNRS and Université de Nantes, \\ 2 rue de la Houssinière, 44322 Nantes Cedex France. \\ and \\ J. Nourrigat, LMR EA 4535 and FR CNRS 3399, \\ Université de Reims Champagne-Ardenne, \\ Moulin de la Housse, BP 1039, 51687 REIMS Cedex 2, France.
}

\begin{abstract}
The aim of this paper is to review and compare the spectral properties of (the closed extension of ) $-\Delta+U(V \geq 0)$ and $-\Delta+i V$ in $L^{2}\left(\mathbb{R}^{d}\right)$ for $C^{\infty}$ real potentials $U$ or $V$ with polynomial behavior. The case with magnetic field will be also considered. More precisely, we would like to present the existing criteria for:

- essential selfadjointness or maximal accretivity

- Compactness of the resolvent.

- Maximal inequalities, i.e. the existence of $C>0$ such that, $\forall u \in$ $C_{0}^{\infty}\left(\mathbb{R}^{d}\right)$

$$
\|u\|_{H^{2}\left(\mathbb{R}^{d}\right)}^{2}+\|U u\|_{L^{2}\left(\mathbb{R}^{d}\right)}^{2} \leq C\left(\|(-\Delta+U) u\|_{L^{2}\left(\mathbb{R}^{d}\right)}^{2}+\|u\|_{L^{2}\left(\mathbb{R}^{d}\right)}^{2}\right),
$$

or

$$
\|u\|_{H^{2}}^{2}+\|V u\|^{2} \leq C\left(\|(-\Delta+i V) u\|^{2}+\|u\|^{2}\right) .
$$

Motivated by recent works with X. Pan, Y. Almog and D. Grebenkov ([3, 13, 2, 1), we will actually improve the known results in the case with purely imaginary potential.
\end{abstract}

\section{Introduction}

In this paper, we review and compare the spectral properties of (the closed extension of $)-\Delta+U(U \geq 0)$ and $-\Delta+i V$ in $L^{2}\left(\mathbb{R}^{d}\right)$ and more precisely the criteria for:

- essential selfadjointness or maximal accretivity

- Compactness of the resolvent. 
- Maximal inequalities, i.e. the existence of $C>0$ such that, $\forall u \in C_{0}^{\infty}\left(\mathbb{R}^{d}\right)$,

$$
\|u\|_{H^{2}\left(\mathbb{R}^{d}\right)}^{2}+\|U u\|_{L^{2}\left(\mathbb{R}^{d}\right)}^{2} \leq C\left(\|(-\Delta+U) u\|_{L^{2}\left(\mathbb{R}^{d}\right)}^{2}+\|u\|_{L^{2}\left(\mathbb{R}^{d}\right)}^{2}\right)
$$

or

$$
\|u\|_{H^{2}}^{2}+\|V u\|^{2} \leq C\left(\|(-\Delta+i V) u\|^{2}+\|u\|^{2}\right) .
$$

We will also discuss the magnetic case. In this case the operator reads:

$$
P_{\mathbf{A}, W}=-\Delta_{A}+V:=\sum_{j=1}^{d}\left(D_{x_{j}}-A_{j}(x)\right)^{2}+W(x),
$$

where $\mathbf{A}=\left(A_{1}, \ldots, A_{d}\right)$ is a $C^{\infty}$ vector fields on $\mathbb{R}^{d}$ and the maximal regularity is expressed in terms of the magnetic Sobolev spaces:

$$
\begin{aligned}
& \|(D-\mathbf{A}) u\|_{L^{2}\left(\mathbb{R}^{d}, \mathbb{C}^{d}\right)}^{2}+\sum_{j, \ell}\left\|\left(D_{j}-A_{j}\right)\left(D_{\ell}-A_{\ell}\right) u\right\|_{L^{2}\left(\mathbb{R}^{d}\right)}^{2}+\||W| u\|_{L^{2}\left(\mathbb{R}^{d}\right)}^{2} \\
& \quad \leq C\left(\left\|P_{\mathbf{A}, W} u\right\|_{L^{2}\left(\mathbb{R}^{d}\right)}^{2}+\|u\|_{L^{2}\left(\mathbb{R}^{d}\right)}^{2}\right),
\end{aligned}
$$

The question of analyzing $-\Delta+i V$ or more generally $P_{\mathbf{A}, i V}:=-\Delta_{A}+i V$ appears in many situations [2, 3, 1]. It seems therefore useful to present in a unified way, what is known on the subject in the selfadjoint case and try to go further in the accretive case, where much less is known. If we assume that the potential $V$ is $C^{\infty}$, we know that the operator is essentially selfadjoint starting from $C_{0}^{\infty}\left(\mathbb{R}^{d}\right)$ in the first situation and maximally accretive in the second case. Hence in the two cases the closed operator in consideration is uniquely defined by its restriction to $C_{0}^{\infty}$.

At least for the selfadjoint case, the subject has a long story, in which T. Kato and his school plays an important role. We refer to [36] for a rather complete presentation with an exhaustive list of reference. One should also mention the work of Avron-Herbst-Simon (1978) [5] which popularizes the basic questions on the subject and in particular the magnetic bottles.

For the compactness of the resolvent, outside the easy case when $U \rightarrow+\infty$, the story starts around the eighties with the treatment of instructive examples (Simon [35], Robert [29]) and in the case with magnetic field [5] (the simplest example being for $d=2$ and $U=0$, when $B(x) \rightarrow+\infty)$. In the polynomial case, many results are deduced as a byproduct of the analysis in Helffer-Nourrigat [15], at least in the case when $V$ is a sum of square of polynomials. Using Kohn's type inequality, B. Helffer and A. Morame (Mohamed) [14] (1988) obtain more general results which can be combined with the analysis of A. Iwatsuka [19] (1986). Another family of results using the notion of capacity can be found in [22, 21] (see references therein).

T. Kato proves for example the inequality

$$
\|\Delta u\|_{L^{1}}+\|U u\|_{L^{1}} \leq 3\|(-\Delta+U) u\|_{L^{1}}, \forall u \in C_{0}^{\infty}\left(\mathbb{R}^{d}\right),
$$

under the condition that $U \geq 0$ and $U \in L_{l o c}^{1}$.

The generalization to the $L^{p}(p>1)$ is only possible under stronger conditions on $U$. We will mention some of these results but will focus on the $L^{2}$ estimates which 
are some times easier to obtain. In the case, when $U(x)=\sum_{\ell} U_{\ell}(x)^{2}$, the maximal $L^{2}$ estimate is obtained as a byproduct of the analysis of the hypoellipticity (see Hörmander [17], Rothschild-Stein [30] and the book Helffer-Nourrigat [16] (including polynomial magnetic potentials)). This was then generalized to the case when $V$ is a positive polynomial by J. Nourrigat in an unpublished paper [27] circulating in the nineties and used in the PHD of D. Guibourg [11, 12] defended in 1992, which considers the case when the electric potential $U \geq 0$ and the magnetic potential A are polynomials (one chapter treats a more general situation). In his thesis Zhong (1993) proves the same result by showing that $\nabla^{2}(-\Delta+U)^{-1}$ is a Calderon-Zygmund operator. Z. Shen (1995) 31] generalizes the result to the case when $U$ is in the reverse Hölder class $R H_{q}\left(q \geq \frac{d}{2}\right)$, a class which contains the positive polynomials.

Definition 1.1. A locally $L^{q}$ function $\omega$ and strongly positive almost everywhere belongs to $R H_{q}$ if there exists a constant $C>0$ such that for any cube $Q$ in $\mathbb{R}^{d}$

$$
\left(\frac{1}{|Q|} \int \omega^{q} d x\right)^{\frac{1}{q}} \leq C\left(\frac{1}{|Q|} \int \omega d x\right) .
$$

One should also mention the unpublished thesis of Mba-Yébé [23] defended in 1995. Together with the techniques developed by Guibourg, some of his techniques are useful for the improvments presented in the last section.

Z. Shen considers also the case with magnetic fields in 1996 [32]. Further progress are obtained in the thesis of B. Ben Ali [6] (2007), published in [4, 7] and 8]. The methods applied by Shen and Auscher-Ben Ali include the Fefferman-Phong inequalities, the Calderón-Zygmund decompositions, and various techniques of interpolation. We will come back to one of these results in Subsection 2.3.

\section{Kohn's approach}

This approach was mainly used for getting the compactness of the resolvent. Except in a few cases, these estimates do not lead to the maximal regularity but are enough for getting the compactness and we will see that surprisingly they could also be a step for proving $L^{2}$-maximal estimates.

\section{$2.1 \quad$ Self-adjoint case}

Here we mainly refer to [14] (see also [24], [15]). We analyze the problem for the family of operators :

$$
\mathcal{P}_{\mathbf{A}, V}=\sum_{j=1}^{d}\left(D_{x_{j}}-A_{j}(x)\right)^{2}+\sum_{\ell=1}^{p} U_{\ell}(x)^{2} .
$$

Here the magnetic potential $\mathbf{A}(x)=\left(A_{1}(x), A_{2}(x), \cdots, A_{n}(x)\right)$ is supposed to be $C^{\infty}$ and that $U_{j} \in C^{\infty}$. Under these conditions, the operator is essentially self-adjoint 
on $C_{0}^{\infty}\left(\mathbb{R}^{d}\right)$. We note also that it has the form :

$$
\mathcal{P}_{\mathbf{A}, V}=\sum_{j=1}^{d+p} X_{j}^{2}=\sum_{j=1}^{d} X_{j}^{2}+\sum_{\ell=1}^{p} Y_{\ell}^{2},
$$

with

$$
X_{j}=\left(D_{x_{j}}-A_{j}(x)\right), j=1, \ldots, d, Y_{\ell}=U_{\ell}, \ell=1, \ldots, p .
$$

In particular, the magnetic field is recovered by observing that

$$
B_{j k}=\frac{1}{i}\left[X_{j}, X_{k}\right]=\partial_{j} A_{k}-\partial_{k} A_{j}, \text { for } j, k=1, \ldots, d .
$$

Of course, when $U \rightarrow+\infty$, it is well known that the operator has a compact resolvent.(see the argument below).

On the opposite, when $V=0$ and $d=2$ if $B(x)=B_{12} \geq 0$, one immediately deduces from the trivial inequality:

$$
\int B(x)|u(x)|^{2} d x \leq\left\|X_{1} u\right\|^{2}+\left\|X_{2} u\right\|^{2}=\left\langle\mathcal{P}_{\mathbf{A}, V} u \mid u\right\rangle .
$$

that $\lim _{|x| \rightarrow+\infty} B(x)=+\infty$ implies that the operator has a compact resolvent. A typical example is

$$
A_{1}\left(x_{1}, x_{2}\right)=-x_{2} x_{1}^{2}, A_{2}\left(x_{1}, x_{2}\right)=+x_{1} x_{2}^{2} .
$$

In order to treat more general situations, we introduce the quantities:

$$
\check{m}_{q}(x)=\sum_{\ell} \sum_{|\alpha|=q}\left|\partial_{x}^{\alpha} U_{\ell}\right|+\sum_{j<k} \sum_{|\alpha|=q-1}\left|\partial_{x}^{\alpha} B_{j k}(x)\right| .
$$

It is easy to reinterpret this quantity in terms of commutators of the $X_{j}$ 's. When $q=0$, the convention is that

$$
\check{m}_{0}(x)=\sum_{\ell}\left|U_{\ell}(x)\right| .
$$

Let us also introduce

$$
\check{m}^{r}(x)=1+\sum_{q=0}^{r} \check{m}_{q}(x) .
$$

Then the criterion proven by Helffer-Mohamed in 1988 ([14]) is

Theorem 2.1.

Let us assume that there exist $r$ and $C$ such that

$$
\check{m}_{r+1}(x) \leq C \check{m}^{r}(x), \forall x \in \mathbb{R}^{d},
$$

and

$$
\check{m}^{r}(x) \rightarrow+\infty, \text { as }|x| \rightarrow+\infty .
$$

Then, with $U=\sum_{\ell} U_{\ell}^{2}, \mathcal{P}_{\mathbf{A}, U}(h)$ has a compact resolvent. 


\section{Remark 2.2.}

It is shown in 24], that one can get the same result as in Theorem 2.1 under the weaker assumption that

$$
\check{m}_{r+1}(x) \leq C\left[\check{m}^{r}(x)\right]^{1+\delta},
$$

where $\delta=\frac{1}{2^{r+1}-3}(r \geq 1)$. This result is optimal for $r=1$ according to a counterexample by A. Iwatsuka [19] who has exhibited an example of a Schrödinger operator which has a non compact resolvent and such that $\sum_{j<k}\left|\nabla B_{j k}(x)\right|$ has the same order as $\sum_{j<k}\left|B_{j k}\right|^{2}$.

Other generalizations are given in [31] (Corollary 0.11) (see also references therein and [22] for a quite recent contribution including other references).

One can for example replace $\sum_{j} U_{j}^{2}$ by a more general $U$ and the conditions on the $m_{j}$ 's can be reformulated in terms of the variation of $U$ and $B$ in suitable balls. In particular A. Iwatsuka [19] showed that a necessary condition is :

$$
\int_{B(x, 1)}\left(U(x)+\sum_{j<k} B_{j k}(x)^{2}\right) d x \rightarrow+\infty \text { as }|x| \rightarrow+\infty,
$$

where $B(x, 1)$ is the ball of radius 1 centered at $x$.

\subsection{The accretive case : maximal accretivness}

There is a general statement (see for example [15], 3]) about the maximal accretiveness of $\mathcal{P}_{\mathbf{A}, W}:=-\Delta_{A}+W$, when $U \geq 0$.

Theorem 2.3. Consider the magnetic Schrödinger operator $\mathcal{P}:=\mathcal{P}_{\mathbf{A}, W}$ defined on $\mathbb{R}^{d}$ with $\mathbf{A} \in C^{\infty}\left(\mathbb{R}^{d}, \mathbb{R}^{d}\right)$ and $W=U+i V \in C^{\infty}\left(\mathbb{R}^{d}, \mathbb{C}\right)$ such that

$$
U(x) \geq 0
$$

Then the operator $\mathcal{P}$ is maximally accretive. Moreover

$$
\mathcal{P}_{\mathbf{A}, W}=\left(P_{\mathbf{A}, \bar{W}}\right)^{*} .
$$

We extend Theorem 2.1 to the family of operators:

$$
P_{\mathbf{A}, W}=\sum_{j=1}^{d}\left(D_{x_{j}}-A_{j}(x)\right)^{2}+\sum_{\ell=1}^{p} U_{\ell}(x)^{2}+i V(x) .
$$

Here $U=\sum_{\ell=1}^{p} U_{\ell}(x)^{2}$ and $V$ is $C^{\infty}$. We note also that it has the form:

$$
P_{\mathbf{A}, W}=\sum_{j=1}^{d+p} X_{j}^{2}=\sum_{j=1}^{d} X_{j}^{2}+\sum_{\ell=1}^{p} Y_{\ell}^{2}+X_{0},
$$

with

$$
X_{j}=\left(D_{x_{j}}-A_{j}(x)\right), j=1, \ldots, d, Y_{\ell}=U_{\ell}, \ell=1, \ldots, p, X_{0}=i V .
$$


We introduce the new quantity:

$$
\check{m}_{q}(x)=\sum_{\ell} \sum_{|\alpha|=q}\left|\partial_{x}^{\alpha} U_{\ell}\right|+\sum_{j<k} \sum_{|\alpha|=q-1}\left|\partial_{x}^{\alpha} B_{j k}(x)\right|+\sum_{|\alpha|=q-1}\left|\partial_{x}^{\alpha} V\right| .
$$

and keep for $\check{m}^{r}(x)$ the same definition as in previous subsection:

$$
\check{m}^{r}(x)=1+\sum_{q=0}^{r} \check{m}_{q}(x) \text {. }
$$

Then the criterion reads

Theorem 2.4.

Let us assume that there exist $r$ and $C_{0}$ such that

$$
\check{m}_{r+1}(x) \leq C_{0} \check{m}^{r}(x), \forall x \in \mathbb{R}^{d} .
$$

In this case, we say that $(\mathbf{A}, W) \in \mathcal{T}\left(r, C_{0}\right)$.

Then there exist $\delta>0$ and $C_{1}:=C_{1}\left(C_{0}\right)$ such that, $\forall u \in C_{0}^{\infty}\left(\mathbb{R}^{d}\right)$

$$
\left\|\left(\check{m}^{r}(x)\right)^{\delta} u\right\|^{2} \leq C_{1}\left(\left\|P_{\mathbf{A}, W} u\right\|^{2}+\|u\|^{2}\right) .
$$

Remark 2.5. The proof, which was first given in a particular case in [3], will show that we can take $\delta=2^{-r}$ which is in general not optimal. This $\delta$ can indeed be improved when $U=0$ and $\mathbf{A}=0$ (see [1]) but this improvment will not be used in this paper.

Corollary 2.6. Under the same assumptions, if

$$
\check{m}^{r}(x) \rightarrow+\infty, \quad \text { as }|x| \rightarrow+\infty \text {. }
$$

Then $\mathcal{P}_{\mathbf{A}, W}(h)$ has a compact resolvent.

Before entering into the core of the proof, we observe that we can replace $\check{m}^{r}(x)$ by an equivalent $C^{\infty}$ function $\Psi(x)$ which has the property that there exist constants $C_{\alpha}$ and $C>0$ such that :

$$
\begin{aligned}
& \frac{1}{C} \Psi(x) \leq \check{m}^{r}(x) \leq C \Psi(x) \\
& \left|D_{x}^{\alpha} \Psi(x)\right| \leq C_{\alpha} \Psi(x)
\end{aligned}
$$

Indeed, it suffices to replace quantities like $\sum\left|u_{k}\right|$ by $\left(\sum\left|u_{k}\right|^{2}\right)^{1 / 2}$, in the definition (2.13) of $\check{m}_{q}$. The second condition is a consequence of (2.15).

In the same spirit as in Kohn's proof, let us introduce:

\section{Definition 2.7.}

For all $s>0$, we denote by $M^{s}$ the space of $C^{\infty}$ real functions $T$ such that there exists $C_{s}$ such that:

$$
\left\|\Psi^{-1+s} T u\right\|^{2} \leq C_{s}\left(\left\|P_{\mathbf{A}, W} u\right\|\|u\|+\|u\|^{2}\right), \forall u \in C_{0}^{\infty}\left(\mathbb{R}^{d}\right) .
$$


We observe that

$$
U_{\ell} \in M^{1}
$$

and we will show the

\section{Lemma 2.8 .}

$$
i\left[X_{j}, X_{k}\right] \in M^{\frac{1}{2}}, \forall j, k=1, \ldots, d
$$

and

$$
V \in M^{\frac{1}{2}}
$$

Another claim is contained in the

\section{Lemma 2.9.}

If $T$ is in $M^{s}$ and $\left|\partial_{x}^{\alpha} T\right| \leq C_{\alpha} \Psi$ then $i\left[X_{k}, T\right] \in M^{\frac{s}{2}}$, when $|\alpha|=1$ or $|\alpha|=2$.

Assuming these two lemmas, then it is clear that

$$
\Psi(x) \in M^{2^{-r}} .
$$

Lemma 2.9 and (2.20) lead to

$$
\partial_{x}^{\alpha} U_{\ell} \in M^{2^{-|\alpha|}},
$$

and we deduce from Lemmas 2.8 and 2.9 .

$$
\partial_{x}^{\alpha} B_{j k} \in M^{2^{-(|\alpha|+1)}} .
$$

The proof of Theorem 2.4 then becomes easy.

\section{Proof of Lemma 2.8}

We start from the identity (and observing that $X_{j}^{*}=X_{j}$ ) :

$$
\begin{aligned}
& \left\|\Psi^{-\frac{1}{2}}\left[X_{j}, X_{k}\right] u\right\|^{2}=\left\langle\left(X_{j} X_{k}-X_{k} X_{j}\right) u \mid \Psi^{-1}\left[X_{j}, X_{k}\right] u\right\rangle \\
& =\left\langle X_{k} u \mid X_{j} \Psi^{-1}\left[X_{j}, X_{k}\right] u\right\rangle \\
& -\left\langle X_{j} u \mid X_{k} \Psi^{-1}\left[X_{j}, X_{k}\right] u\right\rangle \\
& =\left\langle X_{j} u \mid \Psi^{-1}\left[X_{k}, X_{j}\right] X_{k} u\right\rangle \\
& -\left\langle X_{k} u \mid \Psi^{-1}\left[X_{k}, X_{j}\right] X_{k} u\right\rangle \\
& +\left\langle X_{j} u \mid\left[X_{k}, \Psi^{-1}\left[X_{k}, X_{j}\right]\right] u\right\rangle \\
& -\left\langle X_{k} u \mid\left[X_{j}, \Psi^{-1}\left[X_{k}, X_{j}\right]\right] u\right\rangle \text {. }
\end{aligned}
$$

If we observe that $\Psi^{-1}\left[X_{k}, X_{j}\right]$ and $\left[X_{k}, \Psi^{-1}\left[X_{k}, X_{j}\right]\right]$ are bounded (look at the definition of $\Psi)$, we obtain :

$$
\left\|\Psi^{-\frac{1}{2}}\left[X_{j}, X_{k}\right] u\right\|^{2} \leq C\left(\left\|X_{k} u\right\|^{2}+\left\|X_{j} u\right\|^{2}+\|u\|^{2}\right)
$$

We just observe that

$$
\sum_{j}\left\|X_{j} u\right\|^{2}=\operatorname{Re}\left\langle P_{\mathbf{A}, W} u \mid u\right\rangle
$$

This ends the proof of the first part of the lemma.

For the second part, we start from

$$
\operatorname{Im}\left(\left\langle\Psi^{-1} P_{\mathbf{A}, W} u, Q u\right\rangle\right)=\left\|\psi^{-\frac{1}{2}} Q u\right\|^{2}+\operatorname{Im}\left(\left\langle\Psi^{-1} \sum_{j} X_{j}^{2} u, V u\right\rangle\right),
$$


and observe that

$$
\left\langle\Psi^{-1} X_{j}^{2} u, V u\right\rangle=\left\langle\Psi^{-1} V X_{j} u X_{j} u\right\rangle+\left\langle\left[X_{j}, \Psi^{-1} V\right] X_{j} u \mid u\right\rangle .
$$

Hence

$$
\operatorname{Im}\left\langle\Psi^{-1} X_{j}^{2} u, V u\right\rangle=\operatorname{Im}\left\langle\left[X_{j}, \Psi^{-1} V\right] X_{j} u \mid u\right\rangle .
$$

Then using the property of $V$ and $\Psi$, we get the proof easily.

\section{Proof of Lemma 2.9}

Let $T \in M^{s}$. For each $k$, we can write :

$$
\begin{aligned}
& \left\|\Psi^{-1+\frac{s}{2}}\left[X_{k}, T\right] u\right\|^{2}=\left\langle\Psi^{-1+s}\left(X_{k} T-T X_{k}\right) u \mid \Psi^{-1}\left[X_{k}, T\right] u\right\rangle \\
& =\left\langle\Psi^{-1+s} X_{k} T u \mid \Psi^{-1}\left[X_{k}, T\right] u\right\rangle \\
& -\left\langle\Psi^{-1+s} T X_{k} u \mid \Psi^{-1}\left[X_{k}, T\right] u\right\rangle \\
& =\left\langle\Psi^{-1+s} T u \mid \Psi^{-1}\left[X_{k}, T\right] X_{k} u\right\rangle \\
& -\left\langle X_{k} u \mid \Psi^{-1}\left[X_{k}, T\right] \Psi^{-1+s} T u\right\rangle \\
& +\left\langle T u \mid\left[X_{k}, \Psi^{-2+s}\left[X_{k}, T\right]\right] u\right\rangle \\
& =\left\langle\Psi^{-1+s} T u \mid \Psi^{-1}\left[X_{k}, T\right] X_{k} u\right\rangle \\
& -\left\langle X_{k} u \mid \Psi^{-1}\left[X_{k}, T\right] \Psi^{-1+s} T u\right\rangle \\
& +\left\langle\Psi^{-1+s} T u \mid \Psi^{1-s}\left[X_{k}, \Psi^{-2+s}\left[X_{k}, T\right]\right] u\right\rangle \text {. }
\end{aligned}
$$

We now observe, according to the assumptions of the lemma and the properties of $\Psi$, that $\Psi^{1-s}\left[X_{k}, \Psi^{-2+s}\left[X_{k}, T\right]\right]$ and $\Psi^{-1}\left[X_{k}, T\right]$ are bounded.

So finally we get :

$$
\left\|\Psi^{-\frac{1}{2}}\left[X_{k}, T\right] u\right\|^{2} \leq C\left(\left\|\Psi^{-1+s} T u\right\|^{2}+\left\|X_{k} u\right\|^{2}+\|u\|^{2}\right) .
$$

This ends the proof of the lemma.

\subsection{Nourrigat-Guibourg-Shen results}

The results where first obtained in the polynomial case $(V=0, U \geq 0)$, then for $\mathbf{A}$ and $U \geq 0$ satisfying a condition "à la Helffer-Mohamed" and then with condition of type Reverse-Hölder. We take a version presented in Shen [32] as a consequence of his Theorem 0.9 (see p. 820 lines -13 to -8 ) which in addition refers to [33]. We only write the $L^{2}$ criterion.

Theorem 2.10. For $d \geq 3$, let us assume that $\mathbf{A} \in C^{r+2}\left(\mathbb{R}^{d}\right), U \in C^{r+2}\left(\mathbb{R}^{d}\right)$ and $U \geq 0$ and

$$
\sum_{|\beta|=r+1}\left|\partial_{x}^{\beta} B(x)\right|+\sum_{|\beta|=r+2}\left|\partial_{x}^{\beta} U(x)\right| \leq C \check{m}_{B, U}(x),
$$

where

$$
\check{m}_{B, U}(x)=\sum_{|\beta| \leq r}\left|\partial_{x}^{\beta} B(x)\right|+\sum_{|\beta| \leq r+1}\left|\partial_{x}^{\beta} U(x)\right|+1
$$

Then

$$
\sum_{1 \leq j, \ell \leq d}\left\|\left(D_{x_{j}}-A_{j}(x)\right)\left(D_{x_{\ell}}-A_{\ell}(x)\right) u\right\|_{2} \leq C\left(\left\|P_{\mathbf{A}, U} u\right\|_{2}+\|u\|_{2}\right), \forall u \in C_{0}^{\infty}\left(\mathbb{R}^{d}\right) .
$$


Remark 2.11. As observed in [32], the statement appearing in the thesis of D. Guibourg (Chapter B) is a little weaker. When $d=2$, according to 34 there is no particular difficulty. The assumption $(\mathrm{RH})_{n / 2}$ appearing in [32] needs to change to $(R H)_{p}$ for some $p>1$, and the size estimate for the fundamental solution should have a logarithm. Otherwise, all results should be true.

\section{$3 \quad$ Nilpotent approach}

The basic idea is to start from the maximal hypoellipticity of the Hörmander operator $\mathcal{P}:=\sum_{j} \check{X}_{j}^{2}+\check{X}_{0}$ where the $\check{X}_{j}$ 's are real vector fields satisfying the so called Hörmander condition. When considering the special case of a stratified group $\mathcal{G}=\mathcal{G}_{1} \oplus \cdots \oplus \mathcal{G}_{r}$, with the $\check{X}_{j}$ 's $(j=1, \ldots, k)$ being a basis of $\mathcal{G}_{1}, \check{X}_{0} \in \mathcal{G}_{2}$ and the $\check{X}_{j}(j=0, \ldots, k)$ generating the Lie algebra of rank $r$.

We get for any induced representation $\Pi$ of $\mathcal{G}$ in $\mathcal{H}_{\Pi} \sim L^{2}\left(\mathbb{R}^{k}\right)$ the maximal estimate

$$
\left\|\Pi\left(\check{X}_{0}\right) u\right\|_{\mathcal{H}_{\Pi}}+\sum_{j, \ell=1, \ldots, k}\left\|\Pi\left(\check{X}_{j} \check{X}_{\ell}\right) u\right\|_{\mathcal{H}_{\Pi}} \leq C\|\pi(\mathcal{P}) u\|_{\mathcal{H}_{\Pi}}, \forall u \in \mathcal{S}_{\Pi}
$$

We can then use a variant of Proposition 1.6.1 in [16], to get for any polynomial $V$ with $k$ variables the inequality

$$
\sum_{j, \ell}\left\|\partial_{x_{j} x_{\ell}}^{2} u\right\|+\|V(x) u\| \leq C\|(-\Delta+i V(x)) u\|, \forall u \in C_{0}^{\infty}\left(\mathbb{R}^{k}\right) .
$$

We can indeed find $\mathcal{G}$, a subalgebra $\mathcal{V}$ and $\ell \in \mathcal{G}^{*}$ such that $\Pi$ is unitary equivalent to the representation $\pi_{\ell, \mathcal{V}}$ with

$$
\pi_{\ell, \mathcal{V}}\left(\check{X}_{j}\right)=\partial_{x_{j}} \text { for } j=1, \ldots, k, \pi_{\ell, \mathcal{V}}\left(\check{X}_{0}\right)=i V(x) .
$$

As observed a long time ago, the same approach but looking only at $U(x)=\sum_{j} U_{j}(x)^{2}$ and using the maximal hypoellipticity of $\sum_{j} \check{X}_{j}^{2}$, we obtain

$$
\sum_{j, \ell}\left\|\partial_{x_{j} x_{\ell}}^{2} u\right\|+\|U(x) u\| \leq C\|(-\Delta+U(x)) u\|, \forall u \in C_{0}^{\infty}\left(\mathbb{R}^{d}\right) .
$$

It is then natural to ask if it is true for any polynomial $U \geq 0$ and more generally if we can relax the polynomial condition. Here we refer to Guibourg or Nourrigat 1 [27, 28] for the first point (this is indeed true) and to Shen [31] for the second point. There is indeed a more general class of reverse Hölder potentials:

Definition 3.1. We say that a non negative $\omega$ belongs to the reverse Hölder class if there exists $C$ such that, $\forall x \in \mathbb{R}^{d}, \forall r>0$,

$$
\sup _{y \in B(x, r)} \omega(x) \leq \frac{C}{|B(x, r)|} \int_{B(x, r)} \omega(y) d y .
$$

Note that a non negative polynomial satisfies this condition.

Then other authors work on the subject with the aim of obtaining $L^{p}$ estimates [4, 6]. The case with magnetic fields is always considered.

\footnotetext{
${ }^{1}$ There are actually two different proofs proposed by J. Nourrigat a rather direct one and another based to the analysis of $\sum_{j} \check{X}_{j}^{2}+i \check{X}_{0}$ the difficulty (but this was sometimes treated in [15]) that this operator is no more hypoelliptic.
} 


\section{Maximal estimates for the complex Schrödinger operator with magnetic potentials (non neces- sarily polynomial case)}

\subsection{Main statement}

We consider as before

$$
W=\sum_{\ell} U_{\ell}^{2}+i V
$$

and the associated complex Schrödinger operator $P_{\mathbf{A}, W}$.

Theorem 4.1. If $(\mathbf{A}, W) \in \mathcal{T}\left(r, C_{0}\right)$, there exists $C>0$ such that, for all $u \in$ $C_{0}^{\infty}\left(\mathbb{R}^{d}\right)$ :

$$
\||W| u\|^{2} \leq C\left(\left\|P_{\mathbf{A}, W} u\right\|^{2}+\|u\|^{2}\right) .
$$

\subsection{Hörmander's metrics and partition of unity.}

We introduce, for $t \in[0,1]$ and $x \in \mathbb{R}^{d}$,

$\Phi(x, t)=\sum_{\ell} \sum_{|\alpha| \leq r} t^{|\alpha|+1}\left|\partial_{x}^{\alpha} U_{\ell}(x)\right|+\sum_{j<k} \sum_{|\alpha| \leq r-1} t^{|\alpha|+2}\left|\partial_{x}^{\alpha} B_{j k}(x)\right|+\sum_{|\alpha| \leq r-1} t^{|\alpha|+2}\left|\partial_{x}^{\alpha} V(x)\right|$.

As in Mba-Yébé [23], we introduce a parameter $\mu \geq 1$ to be determined later and which is at the moment arbitrary and we define:

$$
R(x, \mu)=\sup \{t \in[0,1], \quad \Phi(x, t) \leq \mu\}
$$

Proposition 4.2. If $(\mathbf{A}, W) \in \mathcal{T}\left(r, C_{0}\right)$ (see condition (2.15), there exists $C_{2}>1$ such that, for all $t \in(0,1)$, we have the implication:

$$
|y-x| \leq t \Longrightarrow \Phi(y, t) \leq C_{2} \Phi(x, t)+C_{2} t^{r+1} .
$$

Proof. For all $x$ et $u$ in $\mathbb{R}^{d}$ such that $|u|=1$, for all $t$ and $\theta$ such that $0<\theta \leq$ $t \leq 1$, let us introduce:

$$
\Psi(x, u, \theta, t)=\Phi(x+\theta u, t) .
$$

Using Taylor's formula with integral remainder, we can write, if $\theta \leq t$,

$$
\Psi(x, u, \theta, t) \leq C \Phi(x, t)+C R(t),
$$

with

$$
\begin{gathered}
R(t)=t^{r+1} \sum_{\ell} \sum_{|\beta|=r+1} \int_{0}^{\theta}\left|\partial_{x}^{\beta} U_{\ell}(x+\sigma u)\right| d \sigma+\ldots \\
\ldots+C t^{r+1} \sum_{j<k} \sum_{|\beta|=r} \int_{0}^{\theta}\left|\partial_{x}^{\beta} B_{j k}(x+\sigma u)\right| d \sigma+C t^{r+1} \sum_{|\beta|=r} \int_{0}^{\theta}\left|\partial_{x}^{\beta} V(x+\sigma u)\right| d \sigma .
\end{gathered}
$$


Using condition (2.15) and $\theta \leq t \leq 1$, there exists $C>0$ such that

$$
R(t) \leq C \int_{0}^{\theta} \Psi(x, u, \sigma, t) d \sigma+C t^{r+1}
$$

We now apply Gronwall's Lemma and obtain the existence of $C_{2}>0$ such that, for $\theta \leq t \leq 1$

$$
\Psi(x, u, \theta, t) \leq C_{2} \Phi(x, t)+C_{2} t^{r+1} .
$$

This achieves the proof of the proposition.

Proposition 4.3. Let $C_{2}>1$ the constant of Proposition 4.2. Then we have:

$$
|y-x| \leq \frac{R(x, \mu)}{2 C_{2}} \Longrightarrow \frac{1}{2 C_{2}} \leq \frac{R(y, \mu)}{R(x, \mu)} \leq 2 C_{2}
$$

Proof. We apply Proposition 4.2 with $t_{0}=R(x, \mu) \leq 1$. If $|y-x| \leq R(x, \mu)$, we have

$$
\Phi\left(y, t_{0}\right) \leq C_{2}\left(\Phi\left(x, t_{0}\right)+t_{0}^{r+1}\right) \leq 2 C_{2} \mu .
$$

We have indeed $\Phi\left(x, t_{0}\right) \leq \mu$ and $t_{0}^{r+1} \leq 1 \leq \mu$.

Consequently $t_{1}=t_{0} / 2 C_{2}=R(x, \mu) /\left(2 C_{2}\right)$ satisfies $t_{1} \leq 1$ and $\Phi\left(y, t_{1}\right) \leq \mu$. Therefore we get $t_{1} \leq R(y, \mu)$, hence the first inequality above.

If now $|y-x| \leq R(x, \mu) /\left(2 C_{2}\right)$, we deduce $|y-x| \leq R(y, \mu)$, and, permuting the roles of $x$ and $y$, we effectively get

$$
R(y, \mu) \leq 2 C_{2} R(x, \mu) .
$$

This achieves the proof of the proposition.

This proposition shows that the metric defined on $\mathbb{R}^{d}$ by $g_{x}(t)=|t|^{2} / R(x, \mu)^{2}$ $\left(x \in \mathbb{R}^{d}, t \in \mathbb{R}^{d}\right)$, is slowly varying in the sense of Definition 18.4.1 in [18. Moreover, the constant in the definition can be chosen independently of $\mu$. We deduce from Lemma 18.4.4 in [18] the following proposition.

Proposition 4.4. For any $\mu \geq 1$, there exist a sequence of real valued functions $\left(\varphi_{j}\right)$ in $C_{0}^{\infty}\left(\mathbb{R}^{d}\right)$, and a sequence $\left(x_{j}\right)$ in $\mathbb{R}^{d}$, such that:

$$
\begin{gathered}
\sum_{j} \varphi_{j}(x)^{2}=1, \forall x \in \mathbb{R}^{d} . \\
\operatorname{supp} \varphi_{j} \subset B\left(x_{j}, R\left(x_{j}, \mu\right)\right) .
\end{gathered}
$$

- For any multi-index $\alpha$, there exists $\hat{C}_{\alpha}>0$, independent of $\mu$, such that

$$
\sum_{j}\left|\partial^{\alpha} \varphi_{j}(x)\right|^{2} \leq \frac{\hat{C}_{\alpha}}{R(x, \mu)^{2|\alpha|}} .
$$

- There exists $\hat{C}>0$, independent of $\mu$, such that, for $k=1,2$, for any $u$ in $C_{0}^{\infty}\left(\mathbb{R}^{d}\right)$,

$$
\int_{\mathbb{R}^{d}} \frac{|u(x)|^{2}}{R(x, \mu)^{2 k}} d x \leq \hat{C} \sum_{j} \int_{\mathbb{R}^{d}} \frac{\varphi_{j}(x)^{2}|u(x)|^{2}}{R\left(x_{j}, \mu\right)^{2 k}} d x .
$$




\subsection{Proof of Theorem 4.1.}

Just observing that:

$$
\operatorname{Re}<P_{U+i V} f, f>=\|(D-\mathbf{A}) f\|^{2}+\int U|f|^{2} d x
$$

we obtain:

Lemma 4.5. For all $f \in C_{0}^{\infty}\left(\mathbb{R}^{d}\right)$, we have:

$$
\sum_{j}\left\|\left(D_{x_{j}}-A_{j}\right) f\right\|^{2}+\sum_{\ell=1}^{p}\left\|U_{\ell} f\right\|^{2} \leq\left\|P_{\mathbf{A}, W} f\right\|\|f\| .
$$

Proposition 4.6. For any $\mu>1$, let $\left(x_{m}\right)$ be a sequence of points in $\mathbb{R}^{d}$ as in Proposition 4.4. Let $(\mathbf{A}, W) \in \mathcal{T}\left(r, C_{0}\right)$. Then there exist $\mu_{0}>1$ and $C_{3}$ (depending only on $r$ and $C_{0}$ ) such that, for any $m$ such that $R\left(x_{m}, \mu\right) \leq 1 / 2$, and for any $f \in C_{0}^{\infty}\left(\mathbb{R}^{d}\right)$ supported in the ball $B_{m}=B\left(x_{m}, R\left(x_{m}, \mu\right)\right)$ and $\mu \geq \mu_{0}$,

$$
\frac{\mu^{\delta}}{R\left(x_{m}, \mu\right)^{2}}\|f\|+\frac{\mu^{\delta / 2}}{R\left(x_{m}, \mu\right)}\|(D-\mathbf{A}) f\| \leq C_{3}\left\|P_{\mathbf{A}, W} f\right\|
$$

where $\delta$ is the constant given by Theorem 2.4.

Proof.

If $R_{m}:=R\left(x_{m}, \mu\right)$ and

$$
\begin{array}{ll}
V^{(m, l o c)}(y)=R_{m}^{2} V\left(x_{m}+R_{m} y\right), & U_{\ell}^{(m, l o c)}(y)=R_{m} U_{\ell}\left(x_{m}+R_{m} y\right), \\
A_{k}^{(m, l o c)}(y)=R_{m} A_{k}\left(x_{m}+R_{m} y\right), & B_{j k}^{(m, l o c)}(y)=R_{m}^{2} B_{j k}\left(x_{m}+R_{m} y\right) .
\end{array}
$$

If $R_{m} \leq 1$, one verifies that, for any $(\mathbf{A}, W) \in \mathcal{T}\left(r, C_{0}\right)$, the corresponding pair $\left(\mathbf{A}^{(m, l o c)}, W^{(m, l o c)}\right)$ belongs to $\mathcal{T}\left(r, C_{0}\right)$.

If $R_{m} \leq 1 / 2$, we have $\Phi\left(x_{m}, R\left(x_{m}, \mu\right)\right)=\mu$. Applying Proposition 4.2 with $t=R_{m}=R\left(x_{m}, \mu\right) \leq 1$, we have, if $|y| \leq 1$,

$$
\Phi\left(x_{m}, R_{m}\right) \leq C_{2} \Phi\left(x_{m}+R_{m} y, R_{m}\right)+C_{2} \leq C_{2} \check{m}^{r, m, l o c}(y),
$$

where $\check{m}^{r, m, l o c}(y)$ is the function associated, as in (2.13), with the localized operator $P_{\mathbf{A}^{m, l o c}, W^{m, l o c}}$ at the point $x_{m}$. Consequently we have $\mu \leq C_{2} \check{m}^{r, m, l o c}(y)$ for all $y \in \mathbb{R}^{d}$ such that $|y| \leq 1$.

By Theorem 2.4, for all $g \in C_{0}^{\infty}\left(\mathbb{R}^{d}\right)$ with support in $B(0,1)$, we have :

$$
\mu^{\delta}\left(1 / C_{2}\right)^{\delta}\|g\| \leq C_{1}\left(\left\|P_{\mathbf{A}^{m, l o c}, W^{m, l o c}} g\right\|+\|g\|\right)
$$

where $C_{1}$ and $C_{2}$ depend only on $C_{0}$.

Then one can find $\mu_{0}$ and $C_{3}$ with the same properties such that, for $\mu \geq \mu_{0}$ :

$$
\mu^{\delta}\|g\| \leq C_{3}\left\|P_{\mathbf{A}^{m, l o c}, W^{m, l o c}} g\right\| .
$$

If $f$ is supported in $B\left(x_{m}, R\left(x_{m}, \mu\right)\right)$, we apply (4.9) to the function $g(y)=f\left(x_{m}+y R\left(x_{m}, \mu\right)\right)$ and obtain for $\mu \geq \mu_{0}$

$$
\frac{\mu^{\delta}}{R\left(x_{m}, \mu\right)^{2}}\|f\| \leq C_{3}\left\|P_{\mathbf{A}, W} f\right\| \text {. }
$$

Inequality (4.7) leads to (4.8). 


\subsection{End of the proof of Theorem 4.1.}

Let $u \in C_{0}^{\infty}\left(\mathbb{R}^{d}\right)$. For any $\mu \geq 1$, we apply (4.6) and get, distinguishing in the localization formula the $x_{m}$ such that $R\left(x_{m}, \mu\right)>\frac{1}{2}$ from the terms such that $R\left(x_{m}, \mu\right) \leq \frac{1}{2}$,

$$
\begin{gathered}
\int_{\mathbb{R}^{d}}\left[\frac{|u(x)|^{2}}{R(x, \mu)^{4}}+\frac{|(D-\mathbf{A}) u(x)|^{2}}{R(x, \mu)^{2}}\right] d x \leq C\left(\|u\|^{2}+\|(D-\mathbf{A}) u\|^{2}\right)+R, \\
R=C \sum_{R\left(x_{m}, \mu\right) \leq 1 / 2} \frac{\left\|\varphi_{m} u\right\|^{2}}{R\left(x_{m}, \mu\right)^{4}}+\frac{\left\|(D-\mathbf{A})\left(\varphi_{m} u\right)\right\|^{2}}{R\left(x_{m}, \mu\right)^{2}} .
\end{gathered}
$$

Here we have also used (4.5) for the control of commutators. If $\mu \geq \mu_{0}$ with $\mu_{0}$ large enough, for any $m$ such that $R\left(x_{m}, \mu\right) \leq 1 / 2$, we apply Proposition 4.6 to the function $f=\varphi_{m} u$ and obtain:

$$
\begin{gathered}
R \leq C \mu^{-2 \delta} \sum_{R\left(x_{m}, \mu\right) \leq \frac{1}{2}}\left\|P_{\mathbf{A}, W}\left(\varphi_{m} u\right)\right\|^{2} \\
\leq C \mu^{-2 \delta}\left\|P_{\mathbf{A}, W} u\right\|^{2}+C \mu^{-2 \delta} \sum_{m}\left[\left\|\nabla \varphi_{m} \cdot(\nabla-i \mathbf{A}) u\right\|^{2}+\left\|u\left(\Delta \varphi_{m}\right)\right\|^{2}\right] .
\end{gathered}
$$

From (4.5), we deduce:

$$
R \leq C \mu^{-2 \delta}\left\|P_{\mathbf{A}, W} u\right\|^{2}+C \mu^{-2 \delta} \int_{\mathbb{R}^{d}}\left[\frac{|u(x)|^{2}}{R(x, \mu)^{4}}+\frac{|(D-\mathbf{A}) u(x)|^{2}}{R(x, \mu)^{2}}\right] d x .
$$

There exists a possibly new $\mu_{0}$ such that, for $\mu \geq \mu_{0}$,

$$
\int_{\mathbb{R}^{d}}\left[\frac{|u(x)|^{2}}{R(x, \mu)^{4}}+\frac{|(D-\mathbf{A}) u(x)|^{2}}{R(x, \mu)^{2}}\right] d x \leq C\left(\|u\|^{2}+\|(D-\mathbf{A}) u\|^{2}\right)+C \mu^{-2 \delta}\left\|P_{\mathbf{A}, W} u\right\|^{2} .
$$

Using again (4.7), we get:

$$
\int_{\mathbb{R}^{d}}\left[\frac{|u(x)|^{2}}{R(x, \mu)^{4}}+\frac{|(D-\mathbf{A}) u(x)|^{2}}{R(x, \mu)^{2}}\right] d x \leq C\|u\|^{2}+C\left(1+\mu^{-2 \delta}\right)\left\|P_{\mathbf{A}, W} u\right\|^{2} .
$$

Theorem 4.1 follows since $\Phi(x, R(x, \mu)) \leq \mu$ and consequently

$$
R(x, \mu) \sum_{\ell}\left|U_{\ell}(x)\right|+R(x, \mu)^{2} \sum_{j<k}\left|B_{j k}(x)\right|+R(x, \mu)^{2}|V(x)| \leq \mu .
$$

Remark 4.7. The proof of Theorem 4.1 does not give directly the maximal estimates as described in the introduction. But the control of the theorem reduces this question to the analysis for a selfadjoint operator which could be either the magnetic Laplacian $(W=0)$, or the magnetic Schrödinger operator $P_{\mathbf{A}, U}$ or the operator $P_{\mathbf{A}, U+\sqrt{1+V^{2}}}$. For these operators one can for example use Shen's Theorem 2.10 (at least when $d \geq 3$, but see Remark 2.11 for $d=2$ ). Note that to be in $\mathcal{T}\left(r, C_{0}\right)$ is usually not enough except if $\mathbf{A}$ is a polynomial. In this case, one can use the nilpotent approach for getting the maximal regularity of the magnetic Laplacian. Otherwise, one can for example prove the complete maximal estimates under the condition that $(\mathbf{A}, W=0)$ belongs to $\mathcal{T}\left(r^{\prime}, C_{0}^{\prime}\right)$. 


\section{Acknowledgements}

The authors would like to thank J. Camus for the transmission of [11] and Z. Shen for an enlightning clarification [34]. These results were announced at the centennial Kato conference in Tokyo (September 2017). The first author would like the RIMS Kyoto, Y. Nakamura and K. Yajima for their support.

\section{References}

[1] Y. Almog, D. Grebenkov, and B. Helffer. On a Schrödinger operator with a purely imaginary potential in the semiclassical limit. ArXiv 1703-07733 (2017).

[2] Y. Almog and B. Helffer. On the spectrum of non-selfadjoint Schrödinger operators with compact resolvent. Comm. in PDE 40 (8) (2015), pp. 1441-1466.

[3] Y. Almog, B. Helffer, and X. Pan. Superconductivity near the normal state under the action of electric currents and induced magnetic fields in $\mathbb{R}^{2}$. Comm. in Math. Phys. 2010, Vol. 300, Issue 1, pp. 147-184.

[4] P. Auscher and B. Ben Ali. Maximal inequalities and Riesz transform on $L^{p}$ space for Schrödinger operators with non negative potentials. Ann. Inst. Fourier 57 (6) (2007) pp. 1975-2013.

[5] Y. Avron, I. Herbst, and B. Simon. Schrödinger operators with magnetic fields I. General interactions. Duke Math. J. 45 (4), pp. 847-883 (1978).

[6] B. Ben Ali. Inégalités maximales et estimations $L^{p}$ des transformées de Riesz des opérateurs de Schrödinger. Thèse de doctorat de l'université Paris-Sud (2008).

[7] B. Ben Ali. Maximal inequalities and Riesz transform estimates on $L^{p}$ spaces for magnetic Schrödinger operators I. Journal of Functional Analysis 259 (2010) $1631-1672$

[8] B. Ben Ali. Maximal inequalities and Riesz transform estimates on $L^{p}$ spaces for magnetic Schrödinger operators II. Math. Z. (2013) 274:85-116.

[9] G.B. Folland. On the Rothschild-Stein lifting theorem. Comm. in PDE 212 (1977), pp. 165-191.

[10] S. Fournais and B. Helffer. Spectral Methods in Surface Superconductivity. Progress in Non-Linear PDE 77, Birkhäuser (2010).

[11] D. Guibourg. Inégalités maximales pour l'opérateur de Schrödinger, PhD Thesis, Université de Rennes 1, 1992.

[12] D. Guibourg. Inégalités maximales pour l'opérateur de Schrödinger. CRAS 316 (1993), pp. 249-252. 
[13] B. Helffer. Spectral Theory and its Applications. Cambridge University Press (2013).

[14] B. Helffer and A. Mohamed. Sur le spectre essentiel des opérateurs de Schrödinger avec champ magnétique. Ann. Inst. Fourier 38(2), pp. 95-113 (1988).

[15] B. Helffer and F. Nier. Hypoelliptic estimates and spectral theory for FokkerPlanck operators and Witten Laplacians. Springer Lecture Note in Mathematics 1862 (2005).

[16] B. Helffer and J. Nourrigat. Hypoellipticité Maximale pour des Opérateurs Polynômes de Champs de Vecteurs. Progress in Mathematics, Birkhäuser, Vol. 58 (1985).

[17] L. Hörmander. Hypoelliptic second order differential equations. Acta Math. 119 (1967), pp. 147-171.

[18] L. Hörmander. The Analysis of Linear Partial Differential Operators. Volume III, Springer, 1985.

[19] A. Iwatsuka. Magnetic Schrödinger operators with compact resolvent. J. Math. Kyoto Univ. 26, pp. 357-374 (1986).

[20] J. Kohn. Lectures on degenerate elliptic problems. Pseudodifferential operators with applications, C.I.M.E., Bressanone 1977, pp. 89-151 (1978).

[21] V. Kondratiev, V. Mazýa, and M. Shubin. Gauge optimization and spectral properties of magnetic Schrödinger operators. Comm. Partial Differential Equations 34 (2009), no. 10-12, pp. 1127-1146.

[22] V. Kondratiev and M. Shubin. Discreteness of spectrum for the magnetic Schrödinger operators. Comm. Partial Differential Equations 27 (3-4), pp. 477526 (2002).

[23] J.T. Mba Yébé. Réalisation et spectre d'opérateurs de Schrödinger et de KleinGordon avec des potentiels irréguliers. Thèse de Doctorat (Université de Reims) (1995).

[24] M. Meftah. Conditions suffisantes pour la compacité de la résolvante d'un opérateur de Schrödinger avec un champ magnétique. J. Math. Kyoto Univ. 31 (3), pp. 875-880 (1991).

[25] A. Mohamed and J. Nourrigat. Encadrement du $N(\lambda)$ pour un opérateur de Schrödinger avec un champ magnétique et un potentiel électrique. Journal de Mathématiques Pures et Appliquées (9) 70, no 1, pp. 87-99 (1991).

[26] J. Nourrigat. Inégalités $L^{2}$ et représentations de groupes nilpotents. Journal of Functional Analysis 74. October 1987.

[27] J. Nourrigat. Une inégalité $L^{2}$, unpublished manuscript $(\sim 1990)$. 
[28] J. Nourrigat. $L^{2}$ inequalities and representations of nilpotent groups. CIMPA School of Harmonic Analysis. Wuhan (China), April-May 1991.

[29] D. Robert. Comportement asymptotique des valeurs propres d'opérateurs du type Schrödinger à potentiel "dégénéré". J. Math. Pures Appl. (9) 61 (1982), no. 3, pp. 275-300 (1983).

[30] L.P. Rothschild and E. Stein. Hypoelliptic operators and nilpotent groups. Acta Mathematica 137 (1977), pp. 248-315.

[31] Z. Shen. $L^{p}$ estimates for Schrödinger with certain potentials. Ann. Inst. Fourier 45 (1995), pp. 513-546.

[32] Z. Shen. Estimates in $L^{p}$ for magnetic Schrödinger operators. Indiana Univ. Math. J., 45 (1996), pp. 817-841.

[33] Z. Shen. Eigenvalue asymptotics and exponential decay of eigenfunctions for Schrödinger operators with magnetic fields. TAMS 348, pp. 4465-4488 (1996).

[34] Z. Shen. Personal communication. September 2017.

[35] B. Simon. Some quantum operators with discrete spectrum but classically continuous spectrum. Ann. Physics 146, pp. 209-220 (1983).

[36] B. Simon. Tosio Kato's work on non-relativistic quantum mechanics. ArXiv October 2017.

[37] J. Zhong. The Sobolev estimates for some Schrödinger type operators. Math. Sci. Res. Hot-Line 3 (1999), no. 8, pp. 1-48; (and Harmonic Analysis of some Schrödinger type Operators, PhD thesis, Princeton University, 1993). 